Yuchi Jia, Xiaomei Wu*

\title{
In vitro activity of allicin combined with two antibiotics on intestinal Shigella
}

DOI: 10.1515/ii-2017-0152

Received August 22, 2017; accepted August 28, 2017; published online November 28, 2017

\begin{abstract}
Objective: We aimed to evaluate the combined antibacterial effects of allicin in combination with levofloxacin and ceftriaxone on Shigella isolated from the intestinal tract in vitro. Materials and Methods: Using a checkerboard design, broth microdilution assay was used to test the effects of the compounds on the organism. We also determined the MIC of the two groups of antibacterial drugs against 30 strains of Shigella and calculated the fractional inhibitory concentration (FIC) index, to judge the combination effect. Result: After the combined application of allicin and ceftriaxone the MIC decreased significantly. Distribution of the FIC index was as follows: FIC $\leq 0.5$, accounting for $10 \%$; $0.5<$ FIC $\leq 1.0$, accounting for $60 \%$; $1<$ FIC $\leq 2$, accounting for 30\%; FIC $>2$, percentage is zero. After combined application of allicin and levofloxacin, distribution of FIC index was as follows: FIC $\leq 0.5$, ratio is zero; $0.5<$ FIC $\leq 1$, accounting for $56.7 \%$; $1<$ FIC $\leq 2$, accounting for 43.3\%; FIC >2, ratio is zero. Conclusion: After the combined use of ceftriaxone, levofloxacin, and allicin, most of the tests showed synergistic effects and additive effects on Shigella, while some of them showed no correlation and no antagonistic effect.
\end{abstract}

Keywords: allicin, ceftriaxone, levofloxacin, Shigella, combined drug sensitivity

\section{Introduction}

Bacterial infectious diarrhea is still a common disease in China. Shigella is the common pathogen causing infectious diarrhea, and its pathogenic form belongs to a variety of genera. Although infectious diarrhea is self-limiting, the use of antibiotic treatment can relieve symptoms, shorten the duration of the disease (thereby reducing the spread of the disease), and reduce potentially serious complications. However, because of the abuse of antimicrobial agents as well as the ability of Shigella to acquire and disseminate exogenous genes (such as plasmids, transposons, and integrons), the bacteria are susceptible to acquired resistance. Allicin is an oily product produced by splitting alliin with garlic enzyme alliin lyase with broad-spectrum antibacterial effects [1]; it is a nontraditional antibacterial drug. In this study, we investigated the antibacterial activity of allicin in combination with common antibacterial agents (levofloxacin and ceftriaxone) against Shigella in vitro, to evaluate the feasibility of using traditional Chinese medicine in combination with Western medicine for the treatment of infectious diarrhea caused by Shigella.

\section{Materials and Methods}

\subsection{Test Strains}

Thirty strains of Shigella were isolated from diarrhea patients admitted into the intestinal clinic of Tianjin Medical University Second Hospital during the period 2015-2016. All strains were redetermined by PCR.

Department of Infection Research Institute, The Second Hospital of Tianjin Medical University, Tianjin 300211, People’s Republic of China

*Correspondence: Xiaomei Wu, E-mail: qiyue981966715@126.com 


\subsection{Materials, Reagents, and Equipments}

Standard allicin, levofloxacin, and ceftriaxone were purchased from the National Institute for the Control of Pharmaceutical and Biological Products in Beijing, China. Mueller-Hinton (M-H) broth was purchased from Qingdao Hope Bio-Technology Co., Ltd. Blood plate medium and 96 well plates were bought from Tianjin Jin Zhang Science and Technology Development Co., Ltd. Nephelometer was obtained from Becton, Dickinson and Company. Electric thermostatic incubator was from Shanghai CIMO Medical Instrument Manufacturing Co., Ltd.

\subsection{Methods}

\subsubsection{Preparation of Bacterial Suspension}

Five to six colonies of Shigella were isolated and cultured overnight in blood plates, mixed in broth to enrich for 6 hours, making sure that the fluid turbidity after correction by the nephelometer was 0.5 MacFarland; then the bacterial suspension was diluted with $\mathrm{M}-\mathrm{H}$ broth to $1.5 \times 10^{5} \mathrm{CFU} / \mathrm{mL}$ stock.

\subsubsection{Preparation of Antimicrobial Stock Solution}

We referred to the specifications provided by the National Standard Substance Center, aseptic distilled water and $0.1 \mathrm{~mol} / \mathrm{L} \mathrm{NaOH}$ were used as the solvents to dissolve ceftriaxone and levofloxacin to the final concentration of $1280 \mu \mathrm{g} / \mathrm{mL}$. We used anhydrous ethanol as solvent to dissolve allicin to the final concentration of $4096 \mu \mathrm{g} / \mathrm{mL}$.

\subsubsection{Determination of MIC of Allicin Separately}

The MIC of the drug was determined by a microdilution method. The allicin solution was diluted with $\mathrm{M}-\mathrm{H}$ broth at a continuous ratio of nine concentrations: 512, 256, 128, 64, 32, 16, 8, 4, and $2 \mu \mathrm{g} / \mathrm{mL}$, then injected into a sterile 96-well plate, $100 \mu \mathrm{L}$ per well. Subsequently, $100 \mu \mathrm{L}$ of bacterial suspension was added in each well and mixed. Meanwhile, a well with $200 \mu \mathrm{L}$ broth was set as negative control. The plate was cultured for 20 hours at $37^{\circ} \mathrm{C}$ in an electric thermostatic incubator, and the MIC was assessed for allicin as a single drug.

\subsubsection{Sensitivity Test for the Combination Drugs Ceftriaxone}

The levofloxacin and ceftriaxone stock solutions were diluted with $\mathrm{M}-\mathrm{H}$ broth at a continuous ratio of 10 concentrations: $256,128,64,32,16,8,4,2,1$, and $0.5 \mu \mathrm{g} / \mathrm{mL}$. The allicin stock was diluted with M-H broth at a ratio of nine concentrations: 512, 256, 128, 64, 32, 16, 8, 4, and $2 \mu \mathrm{g} / \mathrm{mL}$. Two combinations of allicin and the two antimicrobial agents were added into the 96 -well plates, based on checkerboard design; $50 \mu \mathrm{L}$ of each kind of antibacterial drug was taken, and $1.5 \times 10^{5} \mathrm{CFU} / \mathrm{mL}$ of bacterial suspension $(100 \mu \mathrm{L})$ was added into each well. Each strain was individually provided with an empty line together with $100 \mu \mathrm{L}$ each of ceftriaxone or levofloxacin solution. A negative control was set up by adding sterile broth into a well of each plate. All the plates were cultured for 20 hours at $37^{\circ} \mathrm{C}$ in an electric thermostatic incubator. The MIC values for the single A drug and B drug alone were recorded. Simultaneously, the best combination of the two drugs was selected, and the respective MIC values of A and B combinations were recorded to calculate the FIC index. 


\subsubsection{FIC Index Calculation and Interpretation}

FIC index = ceftriaxone MIC $\mathrm{drug}$ A combined $/ \mathrm{MIC}_{\text {drug A single }}+\mathrm{MIC}_{\text {drug B combined }} / \mathrm{MIC}_{\text {drug B single }}$. FIC $\leq 0.5$ indicates synergistic action; $0.5<$ FIC $\leq 1$ indicates additive effect; $1<$ FIC $\leq 2$ points to indifferent interaction; FIC $>2$ shows antagonistic effect.

\section{Results}

\subsection{MIC Value When Allicin was used Separately}

Allicin in high concentration as a single drug has obvious inhibitory effect against Shigella, the MIC $_{\text {range }}$ being between 128 and $1024 \mu \mathrm{g} / \mathrm{mL}$. The MIC values are shown in Table 1.

\subsection{Distribution of MIC and FIC Values of the Combination of Allicin and Antibacterial Agents against Shigella}

With the combination of levofloxacin, ceftriaxone and allicin, the MIC results of 30 strains of Shigella are shown in Table 2. It can be seen that the $\mathrm{MIC}_{50}$ and $\mathrm{MIC}_{90}$ values of the two drugs are both decreased when compared with the values obtained with the two drugs used alone. This indicates that the antibacterial activity against Shigella increased after the combined use of antibiotics. FIC index distribution shows that the FIC value of allicin combined with levofloxacin ranged from 0.50 to 1.50 , which mainly appears as an additive effect or an indifferent interaction. The FIC value for application of allicin combined with ceftriaxone ranged from 0.375 to 1.50 , mostly appearing as synergistic action or additive effect. There is no antagonism between the two drugs, as shown in Figure 1.

\section{Discussion}

With the widespread use of antibacterial drugs, the increase in bacterial resistance has become a prominent problem in the field of anti infective medicine. Shigella is a common pathogen of infectious diarrhea, and its resistance rate is increasing in recent years. Among the 30 strains of bacteria that were studied in this experiment, $66.7 \%$ of the isolates show resistance to levofloxacin and ceftriaxone. Due to the commonly used

Tab. 1: The MIC of allicin alone against 30 strains of Shigella

\begin{tabular}{lccc}
\hline Drug & MIC $_{\text {range }}(\mu \mathrm{g} / \mathrm{mL})$ & MIC $_{50}(\mu \mathrm{g} / \mathrm{mL})$ & MIC $_{90}(\mu \mathrm{g} / \mathrm{mL})$ \\
\hline Allicin & $128-1024$ & 256 & 512 \\
\hline
\end{tabular}

Tab. 2: The MIC of antimicrobials alone or in combination with allicin against 30 strains of Shigella

\begin{tabular}{lccccc}
\hline Drug & \multicolumn{3}{c}{ Single } & & \multicolumn{2}{c}{ Combination } \\
\cline { 2 - 3 } \cline { 5 - 6 } & MIC $_{50}(\mu \mathrm{g} / \mathrm{mL})$ & MIC $_{\mathbf{9 0}}(\mu \mathrm{g} / \mathrm{mL})$ & & MIC $_{50}(\mu \mathrm{g} / \mathrm{mL})$ & MIC $_{90}(\mu \mathrm{g} / \mathrm{mL})$ \\
\hline Levofloxacin & 128 & 256 & & 64 & 128 \\
Ceftriaxone & 128 & 256 & & 16 & 128 \\
\hline
\end{tabular}




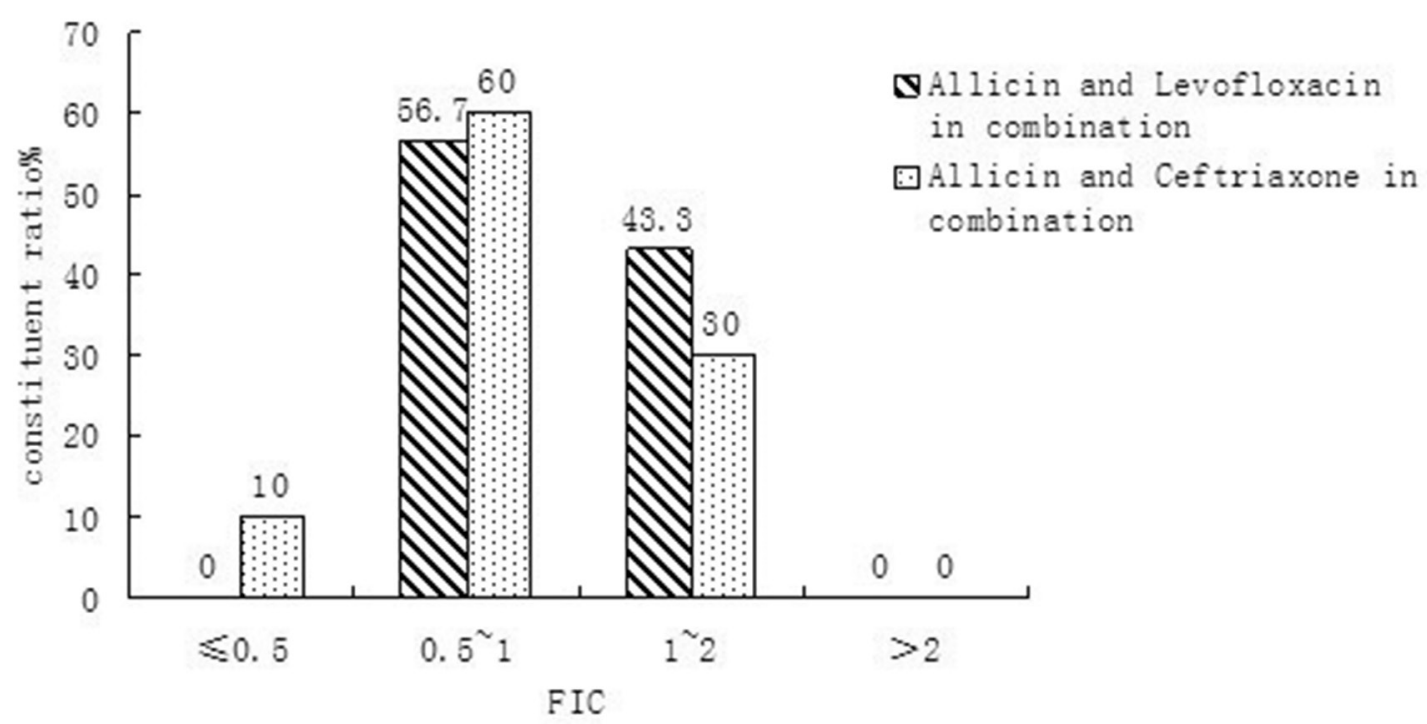

Fig. 1: FIC distribution of antimicrobials in combination with allicin against 30 strains of Shigella.

drugs having limited effects on severe drug-resistant bacteria in outpatient treatment of intestinal diseases, there is a growing tendency to use a combination of antimicrobial agents to control severe infections caused by drug-resistant bacteria. At the same time, this approach can reduce the dose of toxic drugs and reduce adverse reactions. Allicin is a compound isolated from garlic. Its chemical name is 2-allyl sulfo sulfinic acid propyl ester. Allicin is present in garlic in the form of its precursor alliin, which - under the action of alliin lyase - produces allicin with a pungent flavor [2]. It is a natural antibiotic and has good biological performance, immune regulation function, and less adverse reactions, with no antigenicity [3-5]. It can inhibit the common bacteria, fungi, viruses, and even parasites [6]. In this experiment, single use of allicin in vitro on 30 strains of Shigella yielded MIC values of $128-1024 \mu \mathrm{g} / \mathrm{mL}$, indicating that there are limitations in the treatment of Shigella infection with this drug. Coppi et al. [7] found that high-dose allicin has certain amount of toxicity. Hann [8] found that when the concentration of allicin was $>60 \mathrm{mg} / \mathrm{L}$, it could damage mammalian cells in culture. This finding should make the doctor pay attention to the amount of allicin in clinical use. On the other hand, most of the effects are additive actions when allicin is combined with ceftriaxone: their respective $\mathrm{MIC}_{50}$ and $\mathrm{MIC}_{90}$ declined from $50 \%$ to $75 \%$ when compared with monotherapy. By comparison, although the MIC for the combination of allicin and levofloxacin decreased, the experimental bacteria still belonged to the levofloxacin-resistant category, and the effect is mainly additive or independent; however, there was no antagonism between the two drugs. This experiment shows that the clinical application of allicin combined with the antibacterial has certain auxiliary antibacterial effect. Existing studies show that allicin has thioether and allyl groups, which can inhibit enzyme activity by altering cell metabolism, damaging the cell membrane system, and affecting the growth environment of bacteria, thus playing an antibacterial role [9-10]. Its antibacterial mechanism, which is different from that of the general chemical drugs, explains the possibility of using antimicrobial agents as antibiotic sensitizers. With considering the patient's condition, although allicin when used alone has limitations in the treatment of infections, favorable clinical results still can be obtained, based on the in vitro susceptibility test and in vivo test. Meanwhile, due to the immunomodulatory effects of allicin in vivo, it can improve the effect of anti infection treatment. With the further study of the principle of bacteriostasis of garlic extract, as a kind of green safety drug, it will play a more important role in the prevention and cure of disease in the future. 


\section{Acknowledgement: None.}

Conflict of Interest: All authors state no conflict of interest.

Author Contributions: YC Jia conceived the study, performed the experiments, and wrote the paper. XM Wu pointed out design issues and guided corrections. All authors read and approved the manuscript.

\section{References}

[1] Cavallito, C.J., Bailey, J.H., Allicin, the antibacterial principle of Allium sativum. Determination of the chemical structure, J. Am. Chem. Soc., 1944, 5, 1952-1954.

[2] Yan, C.-K., Zeng, F.-D., Recent advances in research of garlic's chemical constituents and theirs pharmacological effects, Chin. J. New Drugs, 2004, 13(8), 688-691.

[3] Ankri, S., Mirelman, D., Antimicrobial properties of allicin from garlic, Microbes Infect., 1999, 1, 125-129.

[4] Bakri, I.M., Douglas, C.W., Inhibitory effect of garlic extract on oral bacteria, Arch. Oral. Biol., 2005, 50, 645-651, DOI: 10.1016/j.archoralbio.2004.12.002

[5] Tsao, S., Yin, M., In vitro activity of garlic oil and four diallyl sulphides against antibiotic-resistant Pseudomonas aeruginosa and Klebsiella pneumoniae, J. Antimicrob. Chemother, 2001, 47, 665-670.

[6] Ruddock, P.S., Liao, M., Foster, B.C., Lawson, L., Arnason, J.T., Dillon, J.A., Garlic natural health products exhibit variable constituent levels and antimicrobial activity against Neisseria gonorrhoeae, Staphylococcus aureus and Enterococcus faecalis, Phytother. Res., 2005, 19(4), 327-334.

[7] Coppi, A., Abinian, M., Mirelman, D., Sinnis P., Antimalarial activity of allicin, a biologically active compound from garlic cloves, Antimicrob. Agents Chemother, 2006, 50(5), 1731-1737.

[8] Hann, G., History, folk medicine, and legendary uses of garlic, In: H.P. Koch, L.D. Lawson (Eds.), Garlic: the science and therapeutic application of Allium sativum L. and related species, Williams and Wilkins, Baltimore, 1996, 37-107.

[9] Kyung, K.H., Antimicrobial properties of allium species, Curr. Opin. Biotechnol., 2012, 23(2), 142-147.

[10] Fujisawa, H., Watanabe, K., Suma, K., Origuchi, K., Matsufuji, H., Seki, T., et al., Antibacterial potential of garlic-derived allicin and its cancellation by sulfhydryl compounds, Biosci. Biotechnol. Biochem., 2009, 73(9), 1948-1955. 